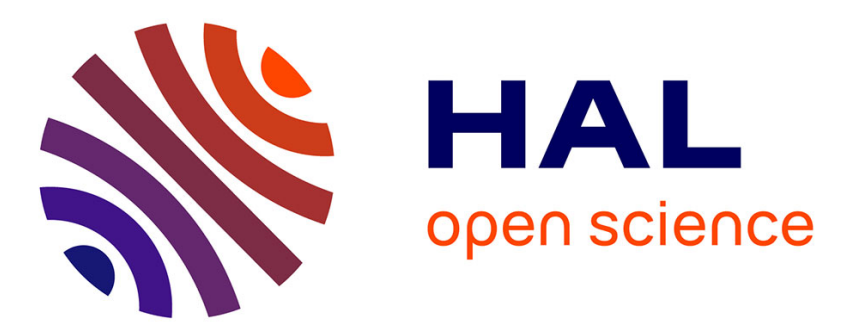

\title{
Perceptions et attitudes liées à la norme ISO 9001 : une analyse auprès de salariés opérationnels
}

\author{
Arnaud Eve, Pierre-Antoine Sprimont
}

\section{To cite this version:}

Arnaud Eve, Pierre-Antoine Sprimont. Perceptions et attitudes liées à la norme ISO 9001: une analyse auprès de salariés opérationnels. Comptabilité - Contrôle - Audit, 2016, 22, pp.27-52. 10.3917/cca.221.0027 . hal-01574343

\section{HAL Id: hal-01574343 https://hal.science/hal-01574343}

Submitted on 6 Oct 2017

HAL is a multi-disciplinary open access archive for the deposit and dissemination of scientific research documents, whether they are published or not. The documents may come from teaching and research institutions in France or abroad, or from public or private research centers.
L'archive ouverte pluridisciplinaire HAL, est destinée au dépôt et à la diffusion de documents scientifiques de niveau recherche, publiés ou non, émanant des établissements d'enseignement et de recherche français ou étrangers, des laboratoires publics ou privés. 


\title{
Arnaud EVE
}

arnaud.eve1@univ-rouen.fr

Maître de conférences

Université de Rouen - IAE

3, avenue Pasteur - CS 46186

76186 ROUEN Cedex 1

\section{Pierre-Antoine SPRIMONT}

pierreantoine.sprimont@univ-rouen.fr

Professeur des Universités

Université de Rouen - IAE

3, avenue Pasteur - CS 46186

76186 ROUEN Cedex 1

\section{Perceptions et attitudes liées à la norme ISO 9001 : une analyse auprès de salariés opérationnels}

Résumé La norme de management de la qualité ISO 9001 est un outil de contrôle. Elle vise la satisfaction du client et l'amélioration continue par la maitrise des processus de l'organisation. Malgré la littérature abondante sur la norme ISO 9001, les effets de sa perception par les salariés opérationnels sont mal connus. L'article explore au moyen d'entretiens et de cartes cognitives, les déterminants de la perception de la norme ISO 9001 et ses effets sur les attitudes de salariés opérationnels. La recherche est menée dans quatre entreprises françaises. L'étude révèle une typologie des perceptions de la norme ISO 9001. Les résultats montrent que des salariés opérationnels perçoivent en majorité favorablement la norme et que des attitudes positives au travail en résultent. Les attitudes influencées par la norme ISO 9001 s'inscrivent dans le contrôle de l'organisation. L'étude souligne la prépondérance du management dans l'appropriation et la perception de la norme ISO 9001.

Mots-clés Contrôle organisationnel ; management de la qualité ; norme ISO 9001 ; attitudes au travail ; cartes cognitives

\section{Perceptions and Attitudes relating to ISO 9001: an Investigation with Operational Personnel}

\begin{abstract}
ISO 9001 International Standard on Quality Management Systems (QMS) is a control tool aimed at customer satisfaction and continual improvement through process management. Despite a substantial literature on ISO 9001 QMS, the consequences of operational employees' perceptions are not well understood. Using interviews and cognitive maps, this article examines determinants of perception of ISO 9001 and its effects on employees' attitudes. The study, which was carried out in four French firms, reveals a typology of perceptions of ISO 9001. The results show that operational workers have mostly positive perceptions of ISO 9001, resulting in positive attitudes in the workplace. The attitudes influenced by the standard contribute to organizational control. The study emphasizes the importance of top management in ISO 9001's adoption and perception.
\end{abstract}

Keywords Organizational Control; Quality Management; ISO 9001; Attitudes in the Workplace; Cognitive Maps 


\section{Perceptions et attitudes liées à la norme ISO 9001 : une analyse auprès de salariés opérationnels}

\section{Introduction}

La norme de management de la qualité ISO 9001 prescrit l'amélioration continue des processus de l'organisation (Dick 2009). Pour Gervais (2009), les systèmes de management, tel l'amélioration de la qualité, complètent les outils du contrôle de gestion que sont par exemple les budgets et les tableaux de pilotage. La norme ISO 9001 qui prescrit le management de la qualité peut donc être interprétée comme un outil de contrôle (Maurand-Valet 2004, p. 33). Cette idée se révèle également dans la contribution de la norme ISO 9001 aux indicateurs de pilotage de l'organisation. La norme promeut l'organisation apprenante et la logique d'amélioration continue. Celles-ci conditionnent l'efficacité des procédures et des processus qui sous-tendent la satisfaction et la fidélisation des clients. On retrouve dans cette chaîne causale d'indicateurs prospectifs l'architecture du Balanced Scorecard (BSC) qui conditionne la performance financière de l'entreprise (Naro et Travaillé 2011). La norme ISO 9001, au même titre que le BSC, contribue donc à une représentation équilibrée de la performance en proposant explicitement de piloter des indicateurs sur des objectifs de satisfaction clients, de respect des procédures et d'apprentissage (Méric 2003).

La norme vise à orienter l'attitude des salariés en faveur de la qualité : les attitudes liées aux principes fondateurs de la norme ISO 9001 (e.g. implication du personnel ${ }^{1}$ ) et les attitudes au sens large (i.e. comportements, croyances et états affectifs). En cela, la norme ISO 9001 soutient la maitrise de l'activité de l'organisation. La norme s'appuie sur le cycle PDCA (Plan, Do, Check, Act) de l'amélioration continue (Pesqueux 2008). Il s'agit du cycle du contrôle à l'origine des travaux du qualiticien William Edwards Deming (Löning et al. 2013). La norme vise la maîtrise des processus de l'organisation par la planification, la mise en œuvre, la mesure et l'analyse corrective des activités. Les quatre phases du cycle PDCA portent le contrôle de gestion et constituent un processus d'apprentissage $^{2}$ (Löning et al. 2013). Sous cet angle, la norme ISO 9001 relève d'une approche cybernétique. Cette même approche cybernétique sous-tend la définition suivante du contrôle: le contrôle se fonde sur la mesure de l'état d'avancement au regard des objectifs à atteindre (Sponem et Chatelain-Ponroy 2013) au moyen d'un feedback informationnel afin de déclencher d'éventuelles actions correctives. Norme ISO 9001 et contrôle partagent donc une même approche cybernétique basée sur une même logique d'amélioration continue. Ainsi, comme le soulignent Bessire, Cappelletti et Pigé (2010, p. 11), «[...] la notion de norme est étroitement associée à celle de contrôle ». La norme ISO 9001 stimule le contrôle de gestion (Cappelletti 2010).

Avec la mesure de la performance financière (le contrôle par les résultats), l'amélioration de la qualité de la gestion constitue le second aspect du contrôle de gestion (le contrôle des comportements) (Meyssonnier 2012). Comme le rappellent Sponem et Chatelain-Ponroy (2013, p. 96), il existe une 
diversité de modes de contrôle pour rendre prévisibles les comportements, regroupés avec le contrôle de gestion dans un ensemble plus vaste que l'on nomme contrôle organisationnel (ou contrôle de l'organisation). Sur ce point, si la norme de management de la qualité ISO 9001 s'inscrit au niveau contrôle de gestion, la qualité apparaît également comme un élément central du contrôle interne et de l'appréhension des risques (cf. travaux de Chemangui 2009; Piot et Kermiche 2009). L'asymétrie informationnelle, l'incomplétude du contrôle et l'opportunisme des acteurs altèrent le processus de contrôle de gestion (Véran 2006). Ainsi, les acteurs déterminent l'efficacité des processus. Il est donc essentiel de se préoccuper de leur comportement. L'humain est au cœur du contrôle organisationnel, autant dans sa vision large de régulation des comportements (e.g. Burlaud et Simon 1997) que dans sa perspective de développement du potentiel humain, selon la vision du contrôle de gestion socioéconomique (cf. Cappelletti 2006). Les attitudes telles la motivation et l'implication (Naro et Salez 2010) constituent un élément central de la performance des organisations. Une meilleure connaissance des attitudes liées à la perception de la norme ISO 9001 est ainsi de nature à renforcer la maîtrise de l'activité de l'organisation. La perception renvoie au processus de traitement des données sensorielles d'un individu dans un contexte donné, auxquelles il confère une signification. Dans le cadre de la norme, il s'agit, par exemple, des documents du système qualité (e.g. les procédures, les imprimés) et du discours du management sur le système qualité.

Notre recherche s'inscrit dans la lignée des travaux sur la perception des outils de contrôle par les salariés (Lyne 1992; Gignon-Marconnet 2003; Macinati 2010). Le budget est par exemple différemment perçu par les directeurs administratifs et financiers et les contrôleurs de gestion. Sponem et Lambert (2010) identifient des perceptions différentes de cet outil de gestion qui influencent plus ou moins la satisfaction des acteurs. Qu'en est-il de la norme ISO 9001 ? Comment est-elle perçue par des salariés opérationnels ? Quelles sont les attitudes liées à cette perception ? A travers ces questions, cet article prolonge la littérature sur la norme ISO 9001 en abordant son influence sur du personnel opérationnel. Ce niveau d'analyse, peu développé par la recherche (Boiral 2012), permettra d'appréhender la façon dont les salariés perçoivent la norme ISO 9001 et quelles sont leurs attitudes face à cet outil de contrôle.

Tout d'abord, l'article présente la conceptualisation de la recherche en commençant par un état des lieux des connaissances sur la norme ISO 9001 puis en précisant le concept de norme de management et le corpus de l'appropriation que nous définissons comme un processus par lequel un usage prévu d'un outil va se trouver rejeté, modifié ou approuvé par l'utilisateur (Martineau 2009). Ensuite, l'investigation empirique est présentée à travers quatre entreprises étudiées, au moyen de la méthodologie des cartes cognitives. Enfin, les résultats de la recherche sont interprétés et discutés. 


\section{La norme ISO 9001 : la nécessaire compréhension de son appropriation au niveau individuel}

La norme ISO 9001 fait état d'une recherche importante. Pour nos travaux, il convient de dresser l'état des connaissances sur la question. Le concept de norme de management dont relève la norme ISO 9001 nécessite d'être discuté. Par ailleurs, la manière de considérer l'appropriation de la norme ISO 9001 doit être examinée. La partie 1 discute l'ensemble de ces points, en commençant par l'état de la recherche sur la norme ISO 9001 dans la section à suivre.

\subsection{Etat de la recherche sur la norme ISO 9001 : un manque de connaissances à combler}

La norme internationale ISO 9001 (version 2008) est la norme de management de la qualité la plus utilisée. C'est une norme générique applicable à tous types d'organisations. Elle prend la forme d'un Système de Management de la Qualité (SMQ). La norme est « certifiable» : pour être reconnues ISO 9001, les organisations font l'objet d'un audit de conformité de leur SMQ à la norme par un organisme tiers et se voient délivrer un certificat. Deux grands thèmes d'investigation résument la recherche sur la norme ISO 9001 (Sampaio et al. 2009, figure 1). D'une part, des travaux étudient l'influence de la norme ISO 9001 sur la performance organisationnelle, financière et managériale. D'autre part, des travaux portent sur les motivations à l'adoption de la norme et son influence sur la certification.

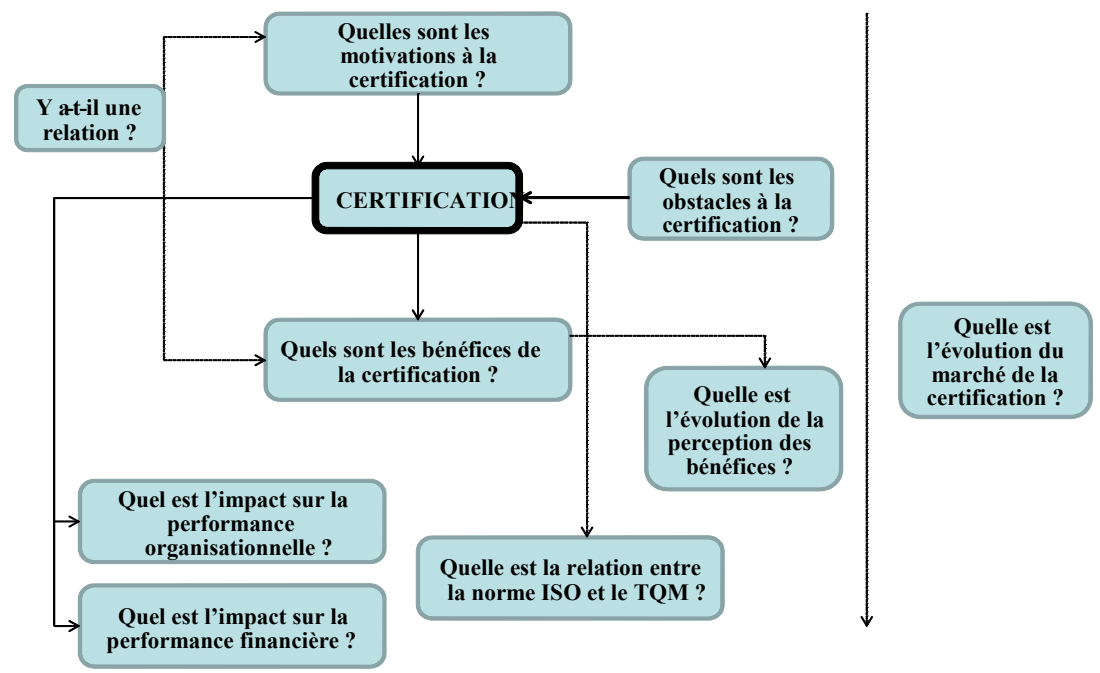

Figure 1: Les questions majeures de recherche sur la norme ISO 9001 (Sampaio et al. 2009)

La norme ISO 9001 ne préconise pas de mesure des résultats économiques qu'elle produit. Sur ce point, la recherche produit des résultats contrastés. Des travaux soutiennent que la norme ISO 9001 améliore la performance financière ${ }^{3}$ de l'organisation (Chow-Chua et al. 2003 ; Corbett et al. 2005 ; 
Sharma 2005). D'autres nuancent cet apport en précisant qu'il s'agit d'une contribution partielle (Sun 2000). Certaines recherches soulignent les coûts importants de mise en œuvre et de maintenance de la norme ISO 9001, qui ne sont pas compensés par les avantages qu'elle procure (Martínez-Costa et Martínez-Lorente 2007). Si le passage de la version 1994 à la version 2000 de la norme ISO 9001 intègre plus largement la philosophie du TQM ${ }^{4}$ (Gotzamani 2005 ; Martínez-Costa et al. 2009), les résultats restent contrastés. Les avantages internes de la norme ISO 9001 aux organisations se caractérisent principalement par des améliorations opérationnelles (e.g. amélioration de la productivité, diminution des non-conformités,...). Les avantages externes de la norme ISO 9001 aux organisations se résument essentiellement à des améliorations marketing (e.g. accès à de nouveaux marchés, satisfaction client,...). Les inconvénients de la norme ISO 9001 aux organisations portent sur son coût de mise en œuvre, sur la rigidification du fonctionnement et la dilution des savoirs individuels dans les connaissances collectives qu'elle entraine. Les entreprises adoptent la norme ISO 9001 selon des motivations internes et/ou externes (Sampaio et al. 2009). Les motivations internes sont principalement centrées sur l'amélioration de l'organisation et de la production. Les motivations externes se réfèrent à la pression des clients, à la promotion de l'image de l'organisation. La recherche montre que le type de motivation à l'adoption de la norme ISO 9001 influence différemment la performance globale de l'organisation (Terziovski et al. 2003 ; Martínez-Costa et al. 2008). L'objet d'analyse de cette littérature est donc l'entreprise. Le niveau «micro» représenté par le salarié opérationnel est négligé. Les conséquences étudiées de la norme portent sur l'entreprise dans son ensemble ; l'influence de la norme ISO 9001 sur l'attitude du salarié est insuffisamment étudiée. Les motivations à la certification et ses conséquences portent sur les décideurs (dirigeant, responsable qualité, managers). La perception du salarié qui vit au quotidien dans un environnement certifié ISO 9001 est peu étudiée. A ce niveau, l'étude de Boiral (2003) fait exception. L'auteur étudie la perception des salariés (managers et employés) sur la norme ISO 9001 et le processus de certification. Boiral identifie trois profils de répondants : les intégrateurs cérémoniels (la norme n'est pas utile pour améliorer le management de la qualité mais c'est un moyen d'améliorer l'image de l'entreprise et d'obtenir de nouveaux contrats), les passionnés de la qualité (la norme favorise le fonctionnement et le contrôle de l'organisation, la réduction des erreurs, la communication client et la promotion de la qualité totale) et les dissidents (la norme est une cage de fer qui limite l'action et dont les avantages commerciaux sont incertains pour compenser ses effets négatifs, c'est-à-dire la bureaucratie). L'étude de Boiral interroge la perception des salariés sur la norme ISO 9001 au niveau de l'entreprise, et non sur l'influence que la norme produit sur leur attitude. De plus, l'approche néo-institutionnelle constitue la principale perspective théorique pour expliquer la rhétorique de la norme, au détriment d'autres modèles explicatifs de la perception individuelle. Des travaux récents traitent de l'adoption de normes ISO de management (Delmas et Pekovic 2013 ; Heras-Saizarbitoria et al. 2013) et de leur influence sur les salariés (Levine et Toffel 2010). Ces recherches n'appréhendent pas les modèles explicatifs de 
l'appropriation, ni l'influence sur des attitudes au sens large. Selon le modèle d'Anderson et al. $(1994)^{5}$, le management de la qualité influence les attitudes au travail. En retour, ces attitudes influencent le management, ce qui constitue un véritable cercle vertueux.

Les publications académiques et professionnelles appellent à un accroissement des connaissances de la relation entre la norme ISO 9001 et l'attitude des salariés. Pour Boiral (2012) la diversité des perceptions dans l'organisation, l'influence de l'utilité de la norme, la mobilisation des employés, etc., restent des thèmes sous-exploités par la littérature. Heras-Saizarbitoria et al. (2013) soulignent un manque important d'études analysant l'adoption de la norme ISO 9001 du point de vue des employés. Recentré sur le salarié agissant au quotidien avec la norme, l'article explore la façon dont le personnel interprète la norme ISO 9001 ; une interprétation que la littérature permet de conceptualiser. La norme de management de la qualité ISO 9001 fait état d'une interprétation large et variée. Il est essentiel de clarifier ces interprétations. L'appropriation de la norme ISO 9001 renvoie à une pluralité de modélisations qu'il est nécessaire d'envisager à l'aune de nos objectifs de recherche. La section à venir discute ces deux points.

\subsection{Conceptualisation de la recherche}

Quel peut être l'effet de la norme ISO 9001 sur l'attitude du personnel ? Différentes visions sont possibles. Par exemple, le management de la qualité met l'accent sur l'implication et la communication du personnel, ce qui pourrait accroitre sa motivation et sa satisfaction. A l'inverse, le management de la qualité qui vise la normalisation des pratiques et le respect des procédures, contribuerait à la passivité des comportements et à décourager l'esprit critique et créatif, et par ricochet réduirait la satisfaction du personnel. Même si les organisations adoptent la même norme (i.e. la norme ISO 9001), elles peuvent l'interpréter de différentes manières, conduisant à des résultats différents (Poksinska 2007). La façon dont la norme est interprétée peut donc conditionner son effet sur l'attitude du personnel. Sur ce point, la littérature propose de multiples définitions de la norme que nous résumons en trois dispositifs (Figure 2). D’une part, la norme apparait comme un dispositif de coordination et de régulation de la qualité et de la performance (e.g. Boiral 2003) portant des règles de fonctionnement et des modèles d'action (e.g. le modèle d'amélioration continue PDCA). D'autre part, la norme apparaît comme un dispositif d'apprentissage et d'innovation (e.g. Haudeville et Wolff 2004). Grâce à la norme, l'organisation capitalise sur son activité, ses pratiques, son expérience, afin d'accroître ses connaissances (réflexion sur le feedback) dans le but de s'améliorer. Puisque la norme permet de faire évoluer l'entreprise vers un nouveau mode d'organisation, il s'agit d'une forme d'innovation organisationnelle. Enfin, en formalisant l'activité, la norme facilite un certain exercice du contrôle (e.g. Burlaud et Zarlowski 2003). La norme s'inscrit dans le processus de vérification des efforts et de réalisation des objectifs. Plus globalement, la norme peut donc être perçue comme un support de gouvernance. A ce niveau, la norme n'est pas vue comme un moyen de maîtrise de 
l'activité de l'organisation (conformément à la perspective anglo-saxonne du contrôle de gestion) mais comme un moyen de vérification, selon la perspective francophone du contrôle de gestion (Bouquin 2005). Au niveau de l'entreprise, trois interprétations de la norme émergent de la littérature : qu'en sera t-il au niveau du salarié ?

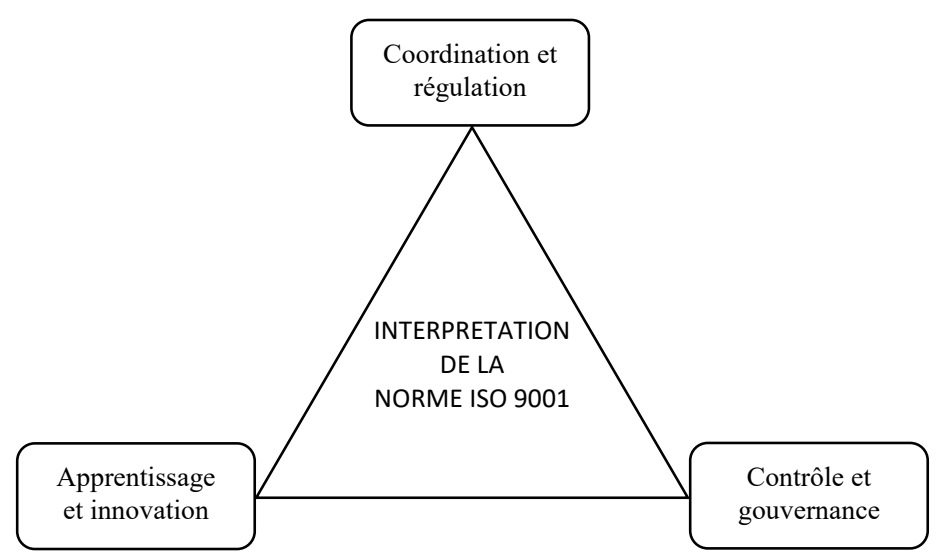

Figure 2 : Les trois dispositifs conceptuels de la norme de management de la qualité ISO 9001

Au niveau de l'entreprise, les normes de management sont d'application volontaire et leur mise en conformité est facultative. Elles fixent des objectifs à atteindre sans imposer de mode de réalisation mais en invitant à la formalisation. Elles s'appliquent à toutes les activités de l'organisation. Elles supposent un droit à la traduction lié à l'usage des acteurs et à leurs intérêts (Igalens et Penan 1994). Les SMQ sont les éléments formalisés (e.g. manuel qualité, procédures, documents) permettant d'orienter l'organisme en matière de qualité. Ils sont conformes à la norme ISO 9001 et prennent en compte les spécificités de chaque organisation. Cette situation permet de conclure à une flexibilité interprétative de la norme. L'influence de la norme sur les salariés dépend de son interprétation et de son appropriation qui différent selon les individus et selon les spécificités de l'environnement dans lequel elle s'établit.

Le lien entre la norme ISO 9001 et l'amélioration de la performance dépend de l'assimilation de la norme (Naveh et Marcus 2004). Cependant, la question de l'appréhension adéquate de la norme ISO 9001 doit être examinée en considération des autres normes présentes dans l'entreprise. Cet environnement normatif est qualifié de « tétranormalisé » ${ }^{6}$. Il renvoie au bombardement des différents types de normes auxquels sont soumises les entreprises (Bessire, Cappelletti et Pigé 2010), source de multiples coûts cachés. Les normes constituent un fond de commerce dont la dynamique concurrentielle les rend incompatibles entre elles (Savall et Zardet 2005). Cette incompatibilité débouche sur une situation de superposition ou d'enchevêtrement des normes générant des incohérences et des conflits (Ebondo Wa Mandzila 2010). L'enchevêtrement de la norme ISO 9001 à d'autres normes dans l'entreprise va introduire de la confusion. Les distorsions générées par la 
tétranormalisation (Bonnet 2009) vont entraver l'appropriation de la norme ISO 9001. La norme ISO 9001 s'inscrit dans un environnement tétranormalisé qui va agir - toute chose égale par ailleurs - sur son appropriation. Sur le thème de l'appropriation, plusieurs modélisations sont envisageables. Des travaux académiques issus de champs disciplinaires variés (e.g. psychologie, systèmes d'information) constituent un terreau fertile pour notre investigation empirique. De cette richesse, nous avons sélectionné plusieurs travaux que nous présentons maintenant.

De Vaujany (2005) propose différentes perspectives de l'appropriation des outils de gestion. La norme ISO 9001 est un outil de gestion différemment interprété selon les individus et selon les contextes. L'outil de gestion sera vu comme un outil de travail selon une perspective rationnelle (la norme régule dans une logique d'efficacité, d'efficience), vu comme un outil de valorisation selon une perspective socio-politique (la norme influence la relation avec les autres) ou vu comme un outil de support d'apprentissage selon une perspective psycho-cognitive (la norme favorise le savoir). La perspective symbolique constitue un quatrième point de vue dans l'appropriation d'un outil de gestion, vu comme un support identitaire vecteur de la construction du sens. La norme peut ainsi porter l'identité des salariés et le sens de leur action au travers de leur préoccupation de la qualité. Cette perspective symbolique et identitaire va également soutenir un autre point de vue dans l'appropriation d'un outil de gestion : celui de la légitimité. A ce niveau, l'appropriation de la norme ISO 9001 dépendra de sa promotion et de sa légitimation (e.g. en mobilisant les symboles) par une institution de l'organisation (e.g. la direction, le responsable qualité). Ainsi l'outil de gestion n'existe que par l'usage et l'interprétation que chacun peut-en faire. Sur ce point, la théorie instrumentale (e.g. Lorino 2002) permet une compréhension de l'outil de gestion. L'outil de gestion est un artefact qui, rencontrant les schèmes d'action de l'utilisateur (la manière dont l'individu conçoit l'utilisation de l'outil), devient un instrument. Suivant l'idée d'Akrich (2006) appliquée à la norme ISO 9001, c'est par sa manière de réagir face à la norme que le salarié lui donne du contenu. La compréhension de l'usage de la norme passe donc par le point de vue des utilisateurs, ouvrant ainsi la voie aux travaux sur l'utilité perçue.

La perspective de l'acceptation (Davis 1989) étudie les déterminants individuels dans l'adoption et l'utilisation d'outils de l'information. En véhiculant l'information par le management des processus, la norme s'inscrit comme un outil de l'information. La perspective de l'acceptation met en avant le rôle de l'utilité et de la facilité d'utilisation perçues dans l'adoption d'outils de l'information. L'utilité perçue (la norme va améliorer la performance au travail) et la facilité d'utilisation perçue (les efforts seront moindres pour utiliser la norme) sont, selon le modèle, les variables d'action à partir desquelles il faut comprendre l'utilisation d'une technologie par un individu. Il s'agit du modèle initial d'acceptation de la technologie (i.e. le modèle TAM : Technology Acceptance Model). Par la suite d'autres recherches vont enrichir ce modèle en étudiant les déterminants de l'utilité et de la facilité perçue (i.e. Venkatesh et Davis 2000 avec le TAM 2 ; Venkatesh et al. 2003 avec le TAM 3). Les 
travaux relatifs au modèle enrichi d'acceptation de la technologie (i.e. Venkatesh et al. 2003) intègrent le corpus théorique de la motivation. A ce niveau, la théorie de l'évaluation cognitive (Deci 1971) suggère que la motivation à agir conditionne l'exercice d'une activité, selon une motivation intrinsèque (la norme permet d'accompagner l'exigence personnelle de qualité) et une motivation extrinsèque (l'entreprise encourage le salarié à suivre la norme). La manière dont le salarié s'approprie la norme détermine son engagement dans celle-ci. Dans ce cas, l'appropriation est fonction de la reconnaissance que le salarié trouve dans la norme elle-même, renforcé dans cette situation par l'entreprise, notamment par un feedback positif'.

Les dispositifs conceptuels de la norme ISO 9001 (i.e. coordination, apprentissage et contrôle) sont essentiellement vus sous l'angle de ses décideurs. Il est essentiel de questionner l'interprétation de ces dispositifs par des opérationnels et la façon dont ils s'approprient la norme dans leur travail quotidien. Ainsi, le corpus vu précédemment constitue un socle conceptuel intégrateur pour appréhender, chez des opérationnels, la perception et les attitudes liées à la norme ISO 9001 (Figure 3). Pour ce faire, quatre organisations certifiées ISO 9001 vont être étudiées. La partie suivante présente ces entreprises et la méthodologie des cartes cognitives.

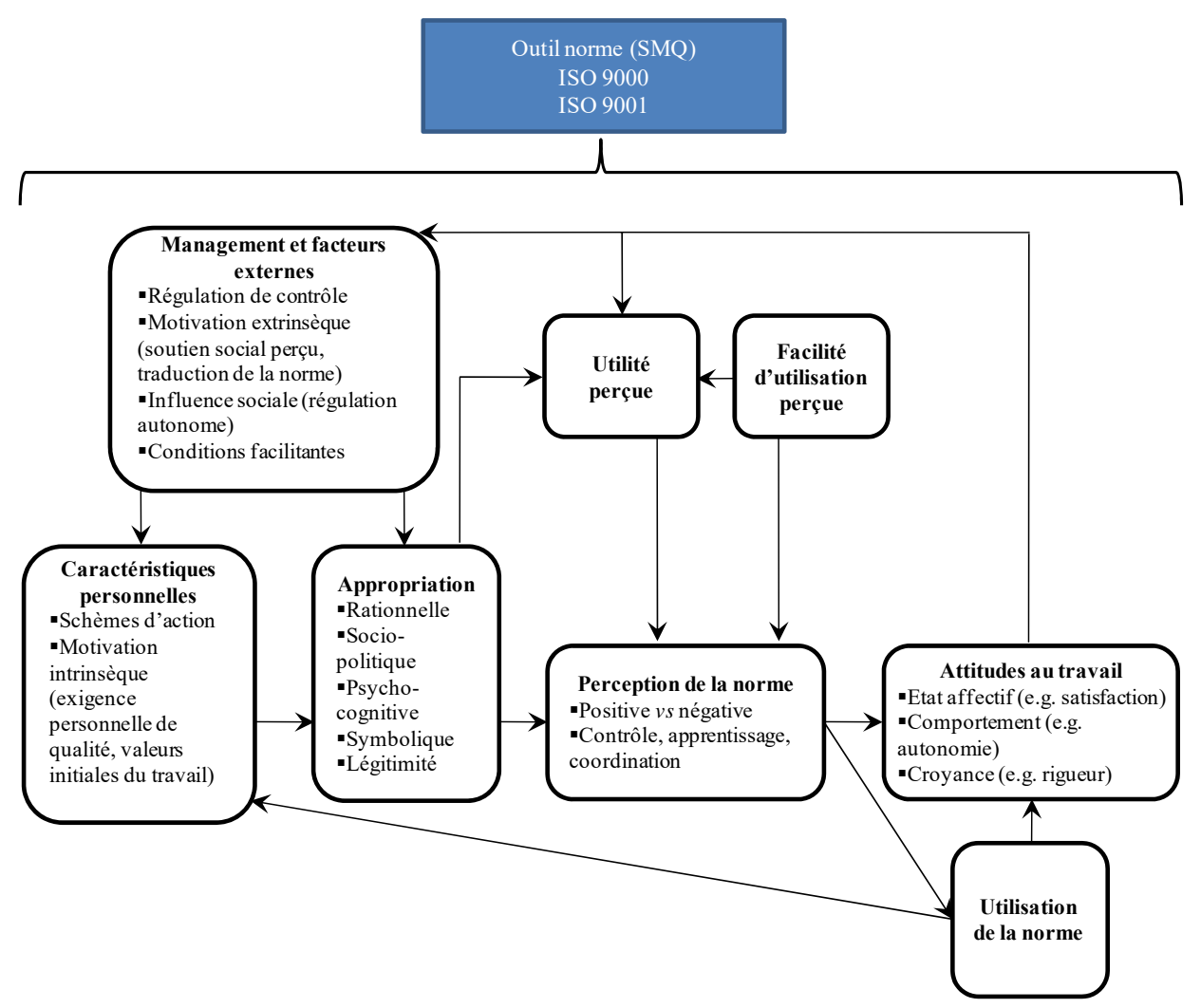

Figure 3 : Socle conceptuel intégrateur 


\section{Investigation empirique}

Dans cette partie, le terrain et la méthodologie sont successivement présentés. Quatre organisations certifiées ISO 9001 (issues de la production, du service et de la santé) ont permis la collecte les données. Outre la variété, le nombre d'organisations ainsi que les effectifs de répondants ont été conditionnés par la saturation des données. Ce principe préside la réalisation des cartes cognitives. Compte-tenu de la préoccupation croissante du management de la qualité dans le secteur de la santé, nous nous sommes rapprochés d'un hôpital privé, dont le service "stérilisation » est certifié. Pour la production industrielle, nous avons sollicité un chantier naval. Ce choix a par ailleurs été motivé par la taille de l'entreprise - environ 400 salariés -, représentative d'un grand nombre d'Entreprises de Tailles Intermédiaires (ETI) en France ${ }^{8}$. Nous avons pu également accéder à une entreprise industrielle de chaudronnerie. Pour le domaine du service, nous nous sommes rapprochés d'un groupe de transport routier de marchandises. Le tableau en annexe 1 présente les caractéristiques des quatre organisations constituant le terrain de l'étude.

Finalement, vingt-sept personnes ont été interviewées (neuf agents, neuf techniciens et neuf cadres). Les agents interviennent en majorité sur site mais certains ont une activité nomade (i.e. les chauffeurs routiers). Les techniciens interviennent sur des aspects administratifs ou pratiques (e.g. maintenance des installations). Les cadres opérationnels interviennent sur des tâches habituelles de gestion et de management. Le tableau en annexe 2 précise les caractéristiques des répondants. L'investigation empirique vise à établir une carte cognitive. La méthodologie des cartes cognitives permet de faire émerger des variables. Elle est particulièrement adaptée pour appréhender les liens d'influence dans un modèle exploratoire. "Une carte cognitive est une représentation graphique de la représentation mentale que le chercheur se fait d'un ensemble de représentations discursives énoncées par un sujet à partir de ses propres représentations cognitives, à propos d'un objet particulier » (Cossette et Audet 2003, p. 34). La carte cognitive est conçue en interrogeant le salarié sur sa perception de la norme ISO 9001 et des liens qu'il établi avec les variables qu'il y associe, mettant au jour d'autres variables faisant l'objet du même questionnement jusqu'à saturation des données. Le résultat final apparaît sous la forme d'une carte cognitive collective agrégée assemblée ( $c f$. figure 4). La carte finale se réfère à un assemblage de cartes individuelles réunies en une seule (e.g. Cossette 2008). Les cartes sont constituées de variables représentées par des bulles et unies par des liens. Les liens sont représentés par des flèches et peuvent former, le cas échéant, des sentiers et des boucles. Conformément au processus d'élaboration des cartes cognitives (e.g. Chaney 2010), des entretiens (semi-dirigés) sont menés pour mettre à jour des variables. Les questions clés des entretiens sont, par exemple : «Qu'estce qu'une norme qualité de type ISO 9001 évoque pour vous ?», "Est-ce qu'une norme qualité de type ISO 9001 influence votre manière d'agir dans votre travail ? ", "Comment ces manières d'agir se traduisent-elles dans votre travail ?». Ces questions permettent d'appréhender les variables 
explicatives et les variables expliquées de la perception de la norme ISO 9001. Les entretiens sont retranscrits et font l'objet d'une analyse textuelle à l'aide du logiciel $Q S R N 6^{\mathscr{C}}$. Les cartes cognitives individuelles sont représentées au moyen du logiciel Decision Explorer ${ }^{\circledR 10}$, en fonction des liens d'influence identifiés au cours de l'analyse textuelle. La partie 3 décrit les résultats de l'étude, qui seront ensuite discutés (partie 4).

\section{Résultats}

Les résultats sont déclinés en trois parties : la perception de la norme ISO 9001, les variables explicatives et les variables expliquées de cette perception. La carte cognitive collective présentée figure 4, représente le résultat final. La discussion finale reviendra sur ces résultats.

\subsection{La perception de la norme ISO 9001}

Les résultats de l'étude mettent en relief cinq perceptions de la norme ISO 9001. Le tableau 1 résume cette typologie.

\begin{tabular}{|c|c|c|c|c|}
\hline $\begin{array}{l}\text { Perception } \\
\text { favorable } \\
\text { (majoritaire) }\end{array}$ & $\begin{array}{l}\text { Perception } \\
\text { défavorable }\end{array}$ & $\begin{array}{c}\text { Perception } \\
\text { distanciée } \\
\text { (potentiel de la norme } \\
\text { jugé incertain et relatif) }\end{array}$ & $\begin{array}{c}\text { Perception } \\
\text { contradictoire }\end{array}$ & Non perception \\
\hline $\begin{array}{c}\text { «la situation serait } \\
\text { moins positive s’il n’y } \\
\text { avait pas la norme ça } \\
\text { amène du positif dans } \\
\text { l'entreprise. On } \\
\text { gagne en qualité de } \\
\text { service et ça a un } \\
\text { impact positif sur les } \\
\text { autres clients » } \\
\text { (technicien, entretien } \\
\left.n^{\circ} 11\right)\end{array}$ & $\begin{array}{c}\text { " la norme, ça sert } \\
\text { strictement à rien, } \\
\text { c'est de la blague » } \\
\text { (agent, entretien n²3) }\end{array}$ & $\begin{array}{c}\text { « la norme c'est un } \\
\text { cadre pour définir une } \\
\text { certaine méthodologie } \\
\text { sur laquelle on peut } \\
\text { s'appuyer pour gagner } \\
\text { en visibilité à } \\
\text { l'extérieur, mais je ne } \\
\text { suis pas intimement } \\
\text { persuadé de la qualité } \\
\text { intrinsèque que cela } \\
\text { apporte au produit } \\
\text { final » } \\
\text { (cadre, entretien } n^{\circ} 6 \text { ) }\end{array}$ & $\begin{array}{l}\text { « la norme ISO 9001 } \\
\text { c'est le gros chaos, [...] } \\
\text { il ne faut pas la } \\
\text { respecter si on veut } \\
\text { avancer [...] la norme } \\
\text { c'est un frein qui a } \\
\text { quand-même comme } \\
\text { avantage de canaliser } \\
\text { les gens dans le même } \\
\text { sens [...] dès que la } \\
\text { norme est respectée, on } \\
\text { retrouve une clarté dans } \\
\text { le travail » } \\
\text { (cadre, entretien } n^{\circ} 7 \text { ) }\end{array}$ & $\begin{array}{c}\text { «on en entend } \\
\text { parler de la norme } \\
\text { mais ça ne me dit } \\
\text { pas grand-chose, } \\
\text { on sait que } \\
\text { l'entreprise est ISO } \\
\text { 9001, sans savoir } \\
\text { ce que c'est» } \\
\text { (agent, entretien } \\
\left.\mathrm{n}^{\circ} 25\right)\end{array}$ \\
\hline
\end{tabular}

Tableau $1:$ typologie des perceptions de la norme ISO 9001

En approfondissant l'analyse, la perception de la norme se décline différemment. Selon les répondants, la norme apparait comme une obligation ou comme un guide d'action volontaire. Une partie des répondants perçoit la norme comme une obligation à laquelle ils doivent satisfaire dans l'exécution de leur travail quotidien. Une autre partie des répondants perçoit la norme comme un guide d'action volontaire :

«la norme c'est comme le code de la route, c'est pour respecter ses engagements, à partir du moment où on est dans la norme, ça oblige à faire un travail que l'on n'aurait pas fait tout de suite » (agent, entretien $\left.\mathrm{n}^{\circ} 21\right)$; «la certification ce n'est pas une obligation, ce n'est pas 
réglementaire, c'est vraiment une démarche spontanée et volontaire, c'est pour améliorer, on fait le choix d'utiliser la norme comme outil de management, d'organisation au sein du groupe» (cadre, entretien $n^{\circ} 2$ )

\subsection{Les variables explicatives de la perception de la norme ISO 9001}

L'étude fait ressortir plusieurs facteurs d'influence de la perception de la norme ISO 9001, regroupés en deux pôles : un pôle management et facteurs externes et un pôle caractéristiques personnelles.

Le management interprète les exigences de la norme. Il conçoit le SMQ appliqué par les opérationnels. Il encadre le fonctionnement quotidien du SMQ et ses phases clés (e.g. audit de certification). Le management influence de manière positive la perception de la norme et favorise l'engagement des salariés. Par ailleurs, le management soutient les salariés dans l'appréhension de la norme :

«l'encadrement nous incite à utiliser la norme »; «je ne connais pas la norme en détail mais ce sont les gens de l'entreprise qui travaillent sur la norme qui me disent les choses à faire dans ce cadre» (agent, entretien $\left.\mathrm{n}^{\circ} 25\right)$; «on travaille avec le responsable qualité sur les axes d'amélioration [...] on nous parle de qualité et on reçoit des modes opératoires du service qualité » (technicien, entretien $\left.n^{\circ} 14\right)$

Cependant, le management est en relation avec une perception moins positive de la norme par les salariés et l'absence de feedback:

«les délégués qualité sont ceux qui mettent en place la norme et nous font faire des conneries»; « on a jamais de point sur ce qui est mis en place grâce à la norme, on a jamais de retour... on fait des points d'information sur les fiches de non-conformité en réunion le matin, mais nous on intervient pas sur la manière de les résoudre, ce serait bien que l'on soit convié à ces réunions... vous demandez à n'importe qui dans l'atelier, les gens savent qu'on est ISO 9001 mais ils sont incapables de vous la décrire » (cadre, entretien $\mathrm{n}^{\circ} 7$ )

Ces verbatim contrastés témoignent de l'importance du management et de sa prépondérance dans l'appropriation et la perception de la norme ISO 9001. Dans l'étude, les facteurs externes se réfèrent à l'influence sociale, aux conditions facilitantes, à la contribution à la mise en œuvre de la norme et, dans une moindre mesure, à la formation et à l'état initial du système qualité. L'influence sociale renvoie à l'influence du groupe social du salarié (e.g. ses collègues d'atelier, ses amis) sur la perception de la norme. Le salarié perçoit la norme conformément à la vision du groupe social. Dans l'étude, les conditions facilitantes s'appréhendent aux travers du processus d'audit. La contribution à la mise en œuvre de la norme traduit la participation à la conception (e.g. rédaction de procédure) du SMQ conforme à la norme ISO 9001. La formation renvoie à l'apprentissage de la qualité dans un 
cursus de formation préalable. L'état initial du système qualité correspond aux caractéristiques du SMQ à l'arrivée du répondant dans le service :

« on sait que l'entreprise est ISO $9001[\ldots]$ en tant qu'ouvrier on ne participe pas aux groupes de résolution de problèmes, quand il y en a, on les règle entre nous dans l'équipe » (agent, entretien $\left.\mathrm{n}^{\circ} 25\right)$; "je trouve que les audits internes sont efficaces, notamment on fait des audits croisés ${ }^{11}$ et c'est efficace » (cadre, entretien $n^{\circ} 9$ ) ; “j'ai été sollicité pour réaliser des procédures du SMQ, j'ai également participé à des audits» (agent, entretien $\mathrm{n}^{\circ} 22$ ); "nous [dans notre fonction] on est formé dans nos études à rédiger des procédures...ce qui nous aide à structurer notre démarche qualité » (cadre, entretien ${ }^{\circ} 2$ ) ; «j'ai repris le système de management de la qualité...et le manuel qualité n'était pas fait comme j'aurais aimé le faire » (cadre, entretien n²)

Les caractéristiques personnelles renvoient à la motivation intrinsèque à s'engager dans la norme, aux valeurs initiales du travail des individus (i.e. la conception personnelle du travail) et à leurs schèmes d'action. Au-delà de leurs spécificités en lien avec la norme, les salariés ont un niveau d'exigence personnelle de la qualité. Les salariés sont animés de valeurs initiales du travail portant sur la norme, la conception personnelle du travail et sa qualité, etc. Les schèmes d'action des individus constituent une autre part déterminante de l'influence des caractéristiques personnelles sur la perception de la norme ISO 9001 :

«il y a des choses qui sont propres à chacun, moi je fonctionne comme ça, je communique énormément»; «avec cette norme [...] il y a une satisfaction personnelle à fournir le travail demandé et à bien le faire» (agent, entretien $\mathrm{n}^{\circ 20}$ ); «Il faut qu’il y ait des normes pour travailler »; «je considère la norme quasiment comme un outil » (agent, entretien $\left.n^{\circ} 21\right)$

\subsection{Les variables expliquées par la perception de la norme ISO 9001}

Les différentes perceptions de la norme ISO 9001 influencent une variété d'attitudes au travail des salariés. Les attitudes sont déclinées par types (états affectifs, comportements et croyances), fonction de la nature des verbatim des répondants.

Les attitudes de type «états affectifs» qui découlent de la perception de la norme ISO 9001 comprennent treize variables: motivation, implication, satisfaction, reconnaissance, scepticisme, confiance, stress, intérêt, fierté, moral, accomplissement, indifférence, mobilisation.

Les attitudes de type «comportements» qui découlent de la perception de la norme ISO 9001 comprennent onze variables : autonomie, communication, passivité, collaboration/travail en équipe, comportement de citoyenneté organisationnelle ( $\mathrm{OCB})$, remise en question, loyauté, retour d'expérience, polyvalence, travail dans le même sens, attention/vigilance. 
Les attitudes de type « croyances » qui découlent de la perception de la norme ISO 9001 comprennent dix variables : performance, amélioration, flicage ${ }^{12}$, qualification/compétence, apprentissage, rigueur, formatage, déresponsabilisation, déqualification, sanction.

La mise en relief de ces attitudes résulte du codage des verbatim. Les exemples suivants illustrent chacune des dimensions de l'attitude :

$>$ Etat affectif de reconnaissance: «la norme ça permet une reconnaissance du service de stérilisation et des personnes parce que nous, en étant au sous-sol, on se sent un peu à l'écart. [...] La norme c'est une reconnaissance de l'entreprise et des acteurs de l'entreprise. [...] Avec la certification c'est une forme de reconnaissance des compétences » (agent, entretien $\left.n^{\circ} 19\right)$;

> Comportement de passivité : «la norme c'est très segmenté, très découpé ce qui altère l'initiative, l'inventivité, le métier au sens noble, l'ouvrage du compagnon » (cadre, entretien $\left.n^{\circ} 6\right)$;

$>$ Croyance de rigueur : « la norme aide à être plus rigoureux. [...] Avec sa méthode, sa rigueur, la norme apporte une sécurité dans le fonctionnement de l'entreprise. [...] La norme ISO 9001 est une aide dans le travail, elle débouche sur de la rigueur » (cadre, entretien n5). 
La carte cognitive collective ci-dessous constitue la représentation graphique du résultat final de la recherche.

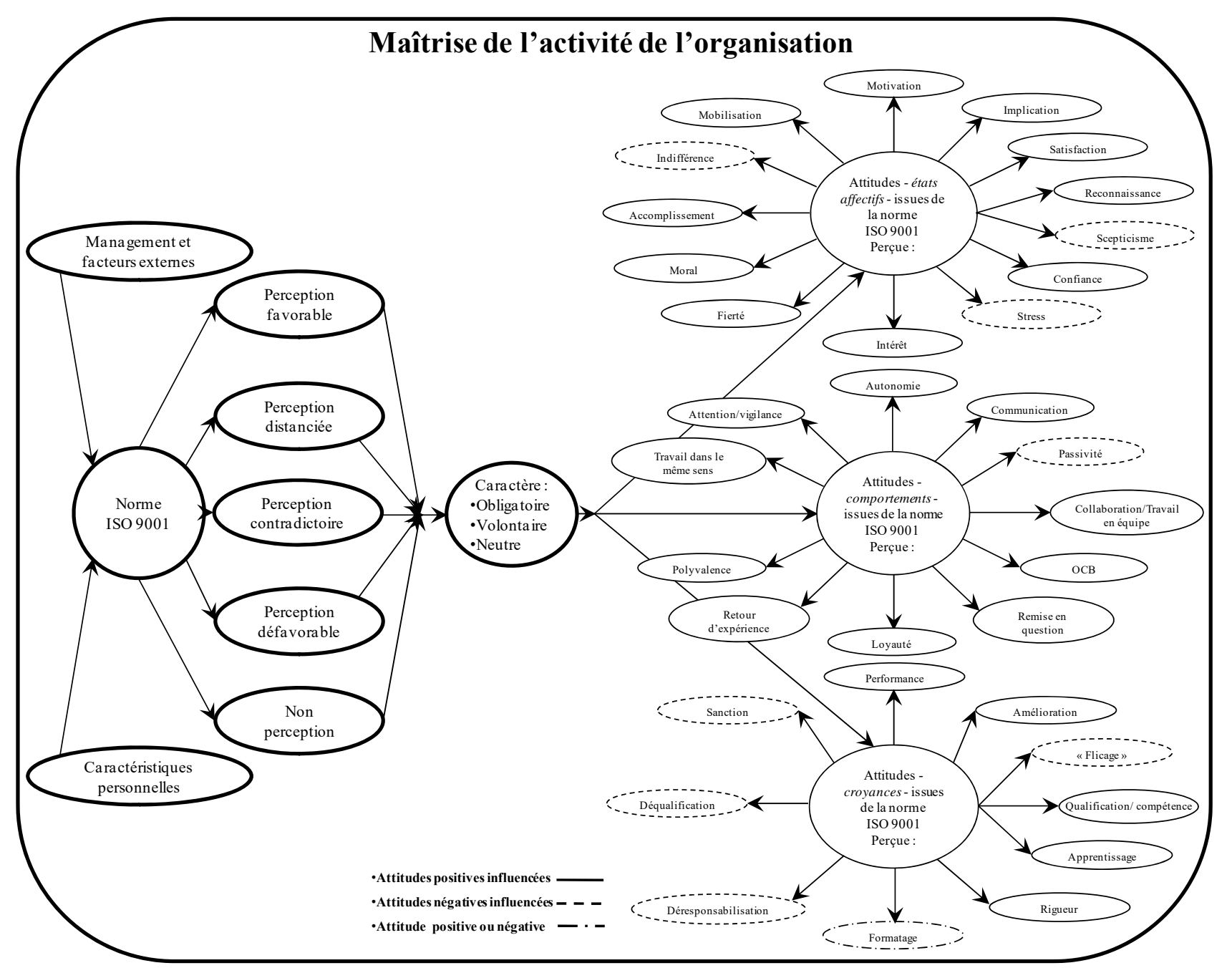

Figure 4 : Carte cognitive collective agrégée assemblée de la perception de la norme ISO 9001

La partie 4 à suivre discute les résultats de l'étude.

\section{Discussion}

Les mécanismes d'appropriation et de perception de la norme ISO 9001 et les attitudes (prégnantes) influencées sont discutés. Ensuite l'influence du management sur la perception de la norme ISO 9001 et, en retour, son influence sur le contrôle de l'organisation sont examinées. 


\subsection{Mécanismes d'appropriation et de perception de la norme ISO 9001}

L'appropriation et la perception de la norme se vérifient à l'aune du cadre d'analyse. L'appropriation de la norme ISO 9001 renvoie aux différentes perspectives mises en évidence dans la littérature. Les perspectives rationnelle et psycho-cognitive ressortent majoritairement. Les perspectives sociopolitique, symbolique et de légitimé sont marginales. Le tableau 2 résume les perspectives d'appropriation issues des résultats de l'étude. Les perspectives d'appropriation de la norme concernent uniquement les perceptions favorable, distanciée et contradictoire de la norme ISO 9001. Pour les perceptions défavorable et non établie (i.e. la non perception), les perspectives d'appropriation ne sont pas vérifiées. Les salariés relevant de ces perceptions ne s'inscrivent dans aucune des cinq perspectives de l'appropriation; bien que les perceptions défavorable et non établie de la norme influencent des attitudes (e.g. l'indifférence). Il est intéressant de noter qu'un même individu peut s'approprier la norme selon plusieurs perspectives, par exemple : perspective rationnelle, perspective de légitimité et perspective psycho-cognitive.

\begin{tabular}{|c|c|c|c|c|}
\hline $\begin{array}{c}\text { Perspective } \\
\text { rationnelle }\end{array}$ & $\begin{array}{c}\text { Perspective socio- } \\
\text { politique }\end{array}$ & $\begin{array}{c}\text { Perspective psycho- } \\
\text { cognitive }\end{array}$ & $\begin{array}{c}\text { Perspective } \\
\text { symbolique }\end{array}$ & $\begin{array}{c}\text { Perspective de } \\
\text { légitimité }\end{array}$ \\
\hline $\begin{array}{c}\text { i.e. la norme est un } \\
\text { outil de travail : } \\
\text { logique d'efficacité }\end{array}$ & $\begin{array}{c}\text { i.e. la norme est un } \\
\text { outil de valorisation, } \\
\text { de rhétorique, } \\
\text { d'influence }\end{array}$ & $\begin{array}{c}\text { i.e. la norme est un } \\
\text { outil d'apprentissage }\end{array}$ & $\begin{array}{c}\text { i.e. la norme est un } \\
\text { outil support de } \\
\text { l'identité et de la } \\
\text { construction du sens } \\
\text { de l'action }\end{array}$ & $\begin{array}{c}\text { i.e. la norme est un } \\
\text { outil de promotion et } \\
\text { de légitimation }\end{array}$ \\
\hline
\end{tabular}

Tableau 2 : les cinq perspectives d'appropriation de la norme ISO 9001

Les modèles explicatifs du cadre d'analyse permettent d'appréhender l'appropriation de la norme ISO 9001, nonobstant de l'environnement tétranormalisé qui se vérifie dans les résultats.

L'utilité perçue et la facilitée d'utilisation perçue de la norme ISO 9001 sont mises en évidence par l'étude pour expliquer l'appropriation de la norme. Les résultats mettent également en relief la motivation intrinsèque et la motivation extrinsèque des salariés à s'engager dans la norme. Le tableau 3 rappelle les différents modèles explicatifs de l'appropriation de la norme ISO 9001 révélés par l'étude. 


\begin{tabular}{|c|c|c|c|}
\hline $\begin{array}{c}\text { Utilité perçue de la } \\
\text { norme ISO 9001 }\end{array}$ & $\begin{array}{c}\text { Facilité d'utilisation } \\
\text { perçue de la norme ISO } \\
\mathbf{9 0 0 1}\end{array}$ & $\begin{array}{c}\text { Motivation intrinsèque } \\
\text { à l'adoption de la } \\
\text { norme ISO 9001 }\end{array}$ & $\begin{array}{c}\text { Motivation extrinsèque } \\
\text { à l'adoption de la } \\
\text { norme ISO 9001 }\end{array}$ \\
\hline $\begin{array}{c}\text { i.e. la norme va } \\
\text { améliorer la performance } \\
\text { au travail }\end{array}$ & $\begin{array}{c}\text { i.e. les efforts seront } \\
\text { moindres pour utiliser la } \\
\text { norme }\end{array}$ & $\begin{array}{c}\text { i.e. la norme permet } \\
\text { d'accompagner } \\
\text { l'exigence personnelle de } \\
\text { qualité }\end{array}$ & $\begin{array}{c}\text { i.e. la nature du } \\
\text { management de } \\
\text { l'entreprise pour } \\
\text { s'engager dans la norme }\end{array}$ \\
\hline
\end{tabular}

Tableau 3 : les modèles explicatifs de l'appropriation de la norme ISO 9001

L'étude permet de souligner que la norme est perçue conformément aux définitions de la littérature, c'est-à-dire: un dispositif de coordination et de régulation, un dispositif d'apprentissage et d'innovation et un dispositif de contrôle et de gouvernance. Le tableau 4 résume les perspectives conceptuelles de la norme issues des résultats de l'étude.

\begin{tabular}{|c|c|c|}
\hline $\begin{array}{c}\text { Dispositif de coordination et } \\
\text { de régulation }\end{array}$ & $\begin{array}{c}\text { Dispositif d'apprentissage et } \\
\text { d'innovation }\end{array}$ & $\begin{array}{c}\text { Dispositif de contrôle et de } \\
\text { gouvernance }\end{array}$ \\
\hline $\begin{array}{l}\text { i.e. la norme porte des règles de } \\
\text { fonctionnement et des modèles } \\
\text { d'action (e.g. le modèle } \\
\text { d'amélioration continue } \\
\text { PDCA) }\end{array}$ & $\begin{array}{c}\text { i.e. grâce à la norme, } \\
\text { l'organisation capitalise sur son } \\
\text { activité, ses pratiques, son } \\
\text { expérience, afin d'accroître ses } \\
\text { connaissances dans le but de } \\
\text { s'améliorer. Puisque la norme } \\
\text { permet de faire évoluer } \\
\text { l'entreprise vers un nouveau } \\
\text { mode d'organisation, il s'agit } \\
\text { d'une forme d'innovation } \\
\text { organisationnelle }\end{array}$ & $\begin{array}{l}\text { i.e. la norme s'inscrit dans le } \\
\text { processus de vérification des } \\
\text { efforts et de réalisation des } \\
\text { objectifs. Plus globalement, la } \\
\text { norme peut donc être perçue } \\
\text { comme un support de } \\
\text { gouvernance }\end{array}$ \\
\hline
\end{tabular}

Tableau 4 : les perspectives conceptuelles de la norme ISO 9001

Il est intéressant de noter que la perception favorable de la norme ISO 9001 n'implique pas nécessairement que les salariés sachent l'interpréter et la relier aux pratiques quotidiennes. Dans ce cas, le répondant déclare ne pas connaître le contenu de la norme ni sa relation avec son travail au quotidien, alors qu'il la juge positive ; ceci alors que l'ensemble des salariés interviewés raccrochent la norme ISO 9001 à l'amélioration de la qualité et la satisfaction du client. Ce point permet de considérer la force des variables explicatives de la perception (i.e. management, facteurs externes et caractéristiques personnelles), au-delà de la considération exclusive des modèles explicatifs de l'appropriation (e.g. l'utilité et la facilité d'utilisation perçue). 


\subsection{Des attitudes variées influencées par la norme ISO 9001}

Dans l'étude, les salariés ont majoritairement une perception favorable de la norme. Ce constat explique que les attitudes influencées par la perception de la norme ISO 9001 soient principalement positives. Parmi les attitudes influencées, la reconnaissance apparaît comme une variable clé. Dans certains cas, son émergence peut s'expliquer par la différence de prestige de la fonction. C'est le cas dans l'organisation de soins et de santé avec la différence de prestige de fonction entre les praticiens (médecins et infirmières) et les salariés de l'activité support de stérilisation. Ainsi, les opérationnels se sentent reconnus grâce à la norme ISO 9001. Dans cette situation, la localisation géographique du service au sous-sol du bâtiment renforce cette explication.

Malgré des attitudes positives influencées par la norme ISO 9001, le stress ressenti au moment de l'audit du SMQ est révélé par l'étude. Si la norme peut constituer une pression au quotidien, la visite de l'auditeur de l'organisme certificateur génère du stress. D'une part, les audits (leviers d'amélioration mettant au jour des axes de progression) génèrent du stress parce qu'ils constituent le moment où l'on connaîtra la réussite aux objectifs de certification. D'autre part, les audits génèrent du stress par les faiblesses éventuelles du SMQ qu'ils peuvent révéler.

Si l'étude soutient la déqualification de l'organisation, cette observation est marginale et non exclusive puisque deux situations de qualification permise par la norme sont déclarées. Par ailleurs, contrairement à la perte d'autonomie au profit du collectif argumentée par les détracteurs de la norme (Segrestin 1996), l'étude suggère que la norme ISO 9001 peut favoriser l'autonomie. Ces points nuancent les inconvénients de la norme ISO 9001 mis en relief par la littérature.

Même si la norme ISO 9001 influence favorablement l'attitude de communication, cette variable s'inscrit dans une perspective négative pour certains salariés. A ce niveau, le management est stigmatisé par le répondant au travers de la norme. D'ailleurs, un autre répondant ayant une vision cette fois-ci - favorable de la norme pointe également un problème de communication dans l'entreprise. Cette situation relativise les travaux affirmant l'influence positive de la norme sur la communication (e.g. Chow-Chua et al. 2003). Les répondants issus du transport routier de marchandises (en toute logique, des salariés opérationnels presqu'exclusivement sur les routes hors de l'entreprise) ne perçoivent pas la communication relative au SMQ. Toutefois, cette situation s'observe également dans un secteur d'activité sédentaire de la production industrielle.

Les résultats mettent en relief la perception double d'une attitude, c'est-à-dire que la même attitude peut être perçue de manière positive ou négative. L'attitude formatage est, dans un cas, perçue positivement et, dans un autre, perçue négativement. La norme va assurer de la continuité et de la fiabilité (i.e. pour la reproductibilité des résultats) dans l'action (formatage positif) ou la norme va restreindre la créativité, l'imagination (formatage négatif). 
Les trois types d'attitudes (états affectifs, comportements, croyances) sont équilibrés (une dizaine de variables constitue chaque type). Si les types d'attitudes états affectif et comportements sont soutenus de manière homogène par des verbatim des quatre entreprises, cette situation diffère pour le type croyances. La moitié des attitudes du type croyances provient d'une organisation du secteur de la production. Bien qu'elles émanent seulement de quelques individus, les variables d'attitudes du type croyances sont particulièrement négatives (e.g. croyance de déqualification, croyance de déresponsabilisation, croyance de sanction influencées par la norme ISO 9001).

Dans les résultats de l'étude, le modèle d'Anderson et al. (1994) se vérifie de façon marginale. Celuici postule que les pratiques de la norme ISO 9001 influencent l'attitude des salariés qui, en retour, va influencer le leadership du management. Sur ce point, le répondant déclare qu'en suivant la norme, il pourra réaliser un travail de qualité. Cette situation conduira son chef à en être satisfait, ce qui devrait le faire évoluer dans l'entreprise.

La situation où la norme ISO 9001, vue comme un ensemble de procédures obligatoires, débouche sur une perception favorable et des attitudes au travail positives est particulièrement intéressante. En effet, elle montre que, bien qu'obligatoire (e.g. sans concertation, négociation ou consultation préalable avec les salariés opérationnels), un outil de gestion peut tout à fait être accepté par le salarié enjoint d'y souscrire. Par exemple, parce qu'il aura la conviction qu'elle peut l'aider dans son travail, le salarié acceptera la norme ISO 9001.

En résumé, dans cette étude, les attitudes influencées par la norme ISO 9001 perçue relèvent principalement de l'état affectif. Elles sont majoritairement positives, quels que soient la catégorie socioprofessionnelle et le secteur d'activité.

\subsection{L'influence du management sur la perception de la norme ISO 9001}

Dans cette recherche, les répondants ont exprimé spontanément la variable management pour expliquer leur perception de la norme.

Sans occulter l'influence des caractéristiques personnelles ou des cadres théoriques majeurs pour expliquer l'interprétation de la norme ${ }^{13}$, l'examen des résultats débouchent sur un constat principal : par son influence, le management est prépondérant dans l'appropriation et la perception de la norme ISO 9001 et les attitudes qui en résultent. Cependant, on constate que l'encadrement ne s'approprie pas systématiquement la norme et que sa perception n'est pas forcément favorable. Cette situation met en relief un paradoxe, dans la mesure où l'encadrement identifie bien les opportunités de la norme, sans qu'il puisse lui-même toujours la mobiliser. Sur ce point, un cadre opérationnel juge la norme favorable à la sensibilisation à la qualité et au leadership. Toutefois, ce répondant dénonce la surfocalisation sur l'établissement d'indicateurs, qu'il estime issue de l'interprétation erronée de la 
norme. L'étude montre bien que l'interprétation de la norme ISO 9001 est un élément crucial (cf. Poksinska, 2007).

Au final, le cadre d'analyse soutient bien les résultats de l'étude. Il valide la séquence selon laquelle le management influence la perception de la norme ISO 9001 qui, à son tour, influence les attitudes au travail (e.g. «l'encadrement nous incite à utiliser la norme »). Notre recherche met par ailleurs en évidence un lien d'influence entre les valeurs initiales du travail et les caractéristiques personnelles, avec la perception de la norme. Si l'on ne peut rejeter l'hypothèse de l'influence de l'organisation sur les valeurs initiales du travail et sur les caractéristiques personnelles, le management est prépondérant pour agir sur la perception de la norme (e.g. «ce sont les gens de l'entreprise qui travaillent sur la norme qui me disent les choses à faire dans ce cadre »). L'étude montre un lien d'influence exprimé par l'ensemble des répondants entre le management et la perception de la norme ISO 9001. Le management confirme son importance dans la perception de la norme et à travers lui, la communication joue un rôle prépondérant pour promouvoir l'amélioration continue.

\subsection{L'influence de la norme ISO 9001 sur le contrôle de l'organisation}

Tels que le montrent les résultats de notre recherche, les attitudes influencées par la perception de la norme ISO 9001 sont majoritairement positives. Cette situation suggère qu'une organisation dotée d'un SMQ selon la norme favorise le contrôle de l'organisation. Appréhender les attitudes influencées par la norme ISO 9001 peut orienter l'action du management vers l'amélioration du SMQ. Cette idée est d'autant plus importante que l'adoption ou le rejet par les acteurs de l'approche processus dans un système de contrôle de gestion, ne sont pas considérés pour le questionnement de la pertinence de l'approche processus (Véran 2006). L'approche processus permettant par essence la maîtrise de l'action, son appropriation n'est pas interrogée. En d'autres termes, on ne cherche pas à savoir si les salariés adoptent ou rejettent la norme ISO 9001, tant l'approche processus est pertinente par définition.

L'étude a mis en relief un ensemble d'attitudes qui s'inscrivent dans le contrôle de l'organisation. Certaines attitudes relevant de l'état affectif correspondent particulièrement à la problématique de mobilisation des salariés ( $c f$. Bouquin 2005). Les résultats de cette recherche montrent que la norme ISO 9001 influence les attitudes de mobilisation, d'implication et de motivation. L'étude a par ailleurs révélé des comportements influencés par la norme ISO 9001. Par exemple, le comportement de travail dans le même sens relève de la convergence des buts visée (cf. Löning et al. 2013). Le comportement déclaré d'autonomie peut s'appréhender à l'aune de la fonction d'autocontrôle recherchée par le contrôle organisationnel. Cette fonction constitue le principe même de l'approche processus au cœur de la norme ISO 9001. L'attitude de comportement de citoyenneté organisationnelle (OCB) influencée par la norme ISO 9001 est particulièrement intéressante dans la recherche en contrôle. Par exemple, 
Piercy et al. (2006) mettent en relief l'OCB comme déterminant de la performance des vendeurs. Les comportements de collaboration, de remise en question et de retour d'expérience influencés par la norme sont également à considérer.

Les attitudes influencées par la norme ISO 9001 relevant des croyances sont plus nuancées. Il y a une part importante d'attitudes négatives. Toutefois, des croyances positives s'inscrivent dans la perspective du contrôle. C'est par exemple le cas avec l'apprentissage (cf. Löning et al. 2013 et Bouquin 2007) et l'amélioration (cf. Bouquin 2007).

Les résultats montrent que, de façon marginale, la norme peut influencer les attitudes de déresponsabilisation, de déqualification, de sanction et de flicage. Si les croyances de sanction et de flicage renvoient à la vision francophone du contrôle de gestion (i.e. un moyen de vérification), les croyances de déresponsabilisation et de déqualification ${ }^{14}$ s'opposent au développement humain. Par ailleurs, on note que l'état affectif d'indifférence et le comportement de passivité ne s'inscrivent pas dans l'idée de maîtrise de l'activité de l'organisation. Ainsi, de façon marginale, la norme ISO 9001 influence des croyances négatives. Cette situation montre bien l'enjeu d'une meilleure appréhension de la norme ISO 9001 à saisir par le management.

Le développement du potentiel humain constitue un enjeu crucial pour les entreprises. L'action du management sur la régulation des attitudes porte un potentiel élevé d'amélioration.

En conclusion, les attitudes influencées par la perception de la norme ISO 9001 s'inscrivent dans le contrôle de l'organisation.

\section{Conclusion}

L'étude souligne que la norme de management de la qualité ISO 9001 est un outil de contrôle de l'organisation, relevant d'une approche cybernétique. A l'appui de la méthode des cartes cognitives (nonobstant des biais potentiels liés à l'approche déclarative de ce type de méthodologie), les résultats montrent les différences de perception de la norme ISO 9001 et les attitudes variées influencées.

Vue comme obligatoire ou comme un guide d'action volontaire, la norme ISO 9001 est en majorité évaluée positivement par les salariés. L'appropriation de la norme débouche sur des attitudes au travail favorables (e.g. satisfaction, reconnaissance, confiance, performance), même si certaines attitudes défavorables sont exprimées (e.g. stress, déqualification). Des attitudes liées à la perception de la norme ISO 9001 s'inscrivent particulièrement dans le contrôle de l'organisation. Par exemple, la mobilisation, l'implication et la motivation.

Le management apparaît comme une variable essentielle dans l'appropriation et la perception de la norme ISO 9001 par les salariés. La séquence selon laquelle le management influence la perception de la norme ISO 9001 qui, à son tour, influence les attitudes au travail, vient enrichir la littérature. Cela 
concerne les effets de la norme ISO 9001 au quotidien dans les organisations, au niveau des salariés opérationnels.

Cette recherche contribue à une meilleure appréhension de l'outil de contrôle norme ISO 9001 et de son appropriation par le personnel opérationnel. La norme est un outil de gestion dont les résultats qu'il produit dépendent de la manière dont il est utilisé. Le management interprète les prescriptions de la norme. Il conçoit le SMQ appliqué par les opérationnels, il encadre le fonctionnement quotidien du SMQ et les phases clés (e.g. audit de certification). Le management revêt une importance particulière dans la mise en œuvre réussie de la norme ISO 9001.

La manière dont la norme ISO 9001 est interprétée et le SMQ conçu conditionnent l'appropriation de la norme. C'est également le cas avec la manière dont la qualité est promue et la manière dont le management encadre le SMQ au quotidien. Cette appropriation par les opérationnels est déterminante pour favoriser des attitudes positives au travail. Ce point s'entend, autant au bénéfice du salarié qu'à celui de l'organisation.

Les variables d'attitude positives résultant de la perception favorable de la norme sont fortement représentées. La norme tend ainsi à produire des effets positifs sur les salariés, profitables à l'organisation (i.e. la performance des salariés). Les variables négatives interpellent le management sur l'interprétation et la mise en œuvre de la norme. L'étude souligne l'importance de la norme pour la satisfaction, la confiance et la reconnaissance des salariés, notamment dans les activités de soutien.

Le management est une variable d'influence incontestable de la perception de la norme ISO 9001. Toutefois, les résultats ne permettent pas d'appréhender le poids des principales variables explicatives (i.e. le management et les caractéristiques personnelles). Par ailleurs, les résultats n'indiquent pas dans quelle mesure le management tente de persuader les salariés de s'engager dans la norme. Une autre perspective de recherche consiste en l'évaluation de la perception de la norme ISO 9001 par l'ensemble des parties prenantes (par exemple les clients). Enfin, les résultats de cette étude nécessitent d'être testés à grande échelle. Ces constats ouvrent la voie à d'autres recherches.

\section{Bibliographie}

- Akrich, M. (2006). La description des objets techniques. In Sociologie de la traduction Textes fondateurs (Eds, Akrich, M., Callon, M., Latour, B.). Paris: Les presses de l'École des Mines de Paris, 159-178.

- Anderson, J. C., Rungtusanatham, M., Schroeder, R. G. (1994). A Theory of Quality Management Underlying the Deming Management Method. Academy of Management Review 19 (3): 472-509. 
- Bessire, D., Cappelletti, L., Pigé, B. (Eds.) (2010). Normes : origines et conséquences des crises. Paris: Economica.

- Boiral, O. (2012). ISO 9000 and Organizational Effectiveness: A Systematic Review. The Quality Management Journal 19 (3): 16-37.

- Boiral, O. (2003). ISO 9000: Outside the Iron Cage. Organization Science 14 (6): 720-737.

- Bouquin, H. (2007). Les fondements du contrôle de gestion - $3^{e}$ édition. Paris: Presses Universitaires de France.

- Bonnet, M. (2009). Assouplir les normes - Le levier de l'harmonisation. Revue Internationale de Psychosociologie 15 (36): 17-20.

- Bouquin, H. (2005). Herméneutique du contrôle. 26ème congrès annuel de l'Association Francophone de Comptabilité, Lille.

- Burlaud, A., Zarlowski, P. (2003). Introduction - Le contrôle externe : quelles modalités pour quels enjeux ? Revue Française de Gestion - Numéro spécial Le contrôle externe modalités et enjeux (147): 9-18.

- Burlaud, A., Simon, C. J. (1997). Le contrôle de gestion. Paris: Éditions La Découverte.

- Cappelletti, L. (2010). Le management de la relation client des professions : un nouveau sujet d'investigation pour le contrôle de gestion. Management \& Avenir (31): 339-363.

- Cappelletti, L. (2006). Le contrôle de gestion socio-économique de la performance : enjeux, conception et implantation. Finance Contrôle Stratégie 9 (1): 135-155.

- Chaney, D. (2010). L'apport des cartes cognitives à l'analyse des représentations mentales. Recherche et Applications en Marketing 25 (2): 93-115.

- Chemangui, M. (2009). Proposition d'une métrique de la qualité de l'audit: expérimentation dans le cadre des relations d'agence internes. Comptabilité - Contrôle - Audit 1: 225-248.

- Chow-Chua, C., Goh, M., Wan, T. B. (2003). Does ISO 9000 Certification Improve Business Performance? International Journal of Quality \& Reliability Management 20 (8): 936-953.

- Corbett, C. J., Montes-Sancho, M. J., Kirsch, D. A. (2005). The Financial Impact of ISO 9000 Certification in the United States: An Empirical Analysis. Management Science 51 (7): 10461059.

- Cossette, P. (2008). La cartographie cognitive vue d'une perspective subjectiviste : mise à l'épreuve d'une nouvelle approche.M@n@gement 11 (3): 259-281.

- Cossette, P., Audet, M. (2003). Qu'est-ce qu'une carte cognitive ? In Cartes cognitives et organisations (Ed, Cossette, P.). Québec ; Paris: Les Éditions de l'ADREG, 31-60.

- Davis, F. D. (1989). Perceived Usefulness, Perceived Ease of Use, and User Acceptance of Information Technology. MIS Quaterly 13 (3): 319-338.

- Deci, E. L. (1971). Effects of Externally Mediated Rewards on Intrinsic Motivation. Journal of Personality And Social Psychology 18 (1): 105-115. 
- Delmas, M. A., Pekovic S. (2013). Environmental Standards and Labor Productivity: Understanding the Mechanisms that Sustain Sustainability. Journal of Organizational Behavior 34: 230-252.

- De Vaujany, F-X. (Ed.). (2005). De la conception à l'usage - Vers un management de l'appropriation des outils de gestion. Colombelles: Editions EMS.

- Dick, G. P.M. (2009). Exploring Performance Attribution - The Case of Quality Management Standards Adoption and Business Performance. International Journal of Productivity and Performance Management 58 (4): 311-328.

- Ebondo Wa Mandzila, E. (2010). Le conflit entre les normes d'audit et de contrôle interne. In Normes : origines et conséquences des crises (Eds, Bessire, D., Cappelletti, L., Pigé, B.). Paris: Economica, 86-92.

- Gervais, M. (2009). Contrôle de gestion - $9^{e}$ édition. Paris: Economica.

- Gignon-Marconnet, I. (2003). Les rôles actuels de la gestion budgétaire en France : une confrontation des perceptions de professionnels avec la littérature. Comptabilité - Contrôle Audit 1: 53-78.

- Gotzamani, K. D. (2005). The Implications of the New ISO 9000: 2000 Standards for Certified Organizations - A Review of Anticipated Benefits and Implementation Pitfalls. International Journal of Productivity and Performance Management 54 (8): 645-657.

- Haudeville, B., Wolff, D. (2004). Enjeux \& déterminants de l'implication des entreprises dans le processus de normalisation. Revue d'Économie Industrielle 108: 21-40.

- Heras-Saizarbitoria, I., Cilleruelo, E., Zamanillo, I. (2013). Adoption of ISO 9000 Practices in Manufacturing Companies: The Perspective of the Shop-Floor Workers. Human Factors and Ergonomics in Manufacturing \& Service Industries 23 (4): 311-321.

- Igalens, J., Penan, H. (1994). La normalisation. Paris: Presses Universitaires de France.

- Levine, D. I., Toffel, M. W. (2010). Quality Management and Job Quality: How the ISO 9001 Standard for Quality Management Systems Affects Employees and Employers. Management Science 56 (6): 978-996.

- Löning, H., Malleret, V., Méric, J., Pesqueux, Y., Solé, A. (2013). Contrôle de gestion - Des outils de gestion aux pratiques organisationnelles - $4^{e}$ édition. Paris: Dunod.

- Lorino, P. (2002). Vers une théorie pragmatique et sémiotique des outils appliqués aux instruments de gestion. Document de recherche DR 02015, ESSEC, Cergy-Pontoise.

- Lyne, S. R. (1992). Perceptions and Attitudes of Different User-Groups to the Role of the Budget, Budget Pressure and Budget Participation. Accounting and Business Research 22 (88): 357-369.

- Macinati, M. S. (2010). NPM Reforms and the Perception of Budget by Hospital Clinicians: Lessons from Two Case-Studies. Financial Accountability \& Management 26 (4): 422-442. 
- Martineau, R. (2009). La mise en usage des outils de gestion par la qualité par les professionnels de santé à l'hôpital : une approche par la théorie instrumentale. Doctorat en sciences de gestion, Tours: Université François Rabelais.

- Martínez-Costa, M., Choi, T. Y., Martínez, J. A., Martínez-Lorente, A. R. (2009). ISO 9000/1994, ISO 9001/2000 and TQM: the Performance Debate Revisited. Journal of Operations Management 27: 495-511.

- Martínez-Costa, M., Martínez-Lorente, A. R., Choi, T. Y. (2008). Simultaneous Consideration of TQM and ISO 9000 on Performance and Motivation: An Empirical Study of Spanish Companies. International Journal of Production Economics 113 (1): 23-39.

- Martínez-Costa, M., Martínez-Lorente, A. R. (2007). A Triple Analysis of ISO 9000 Effects on Company Performance. International Journal of Productivity and Performance Management 56 (5/6): 484-499.

- Maurand-Valet, A. (2004). La norme entre paradoxe et nécessité : une étude du rôle du responsable qualité. Doctorat en sciences de gestion, Montpellier: Université Montpellier II.

- Méric, J. (2003). L'émergence d'un discours de l'innovation managériale - le cas du Balanced Scorecard. Comptabilité - Contrôle - Audit / Numéro spécial: 129-145.

- Meyssonnier, F. (2011). Le contrôle de gestion des services : réflexion sur les fondements et l'instrumentation. Comptabilité - Contrôle - Audit 2: 73-98.

- Naro, G. Travaillé, D. (2011). Le BSC à l'épreuve de l'expérience. De l'assemblage d'un « puzzle » et construction d'un « Lego ». Revue Française de Gestion (211): 65-80.

- Naro, G., Salez, M. (2010). Le contrôle de gestion sociale en quête de fondements théoriques et méthodologiques. In Le contrôle de gestion en mouvement - Etat de l'art et meilleures pratiques (Eds, Berland, N., Simon, F-X.). Paris: Éditions d'Organisation, 127-143.

- Naveh, E., Marcus, A. A. (2004). When does the ISO 9000 Quality Assurance Standard Lead to Performance Improvement? Assimilation and Going Beyond. IEEE Transactions on Engineering Management 51 (3): 352-363.

- Pesqueux, Y. (2008). Planifier, Agir, contrôler : voilà la recette du progrès continu. In Petit bréviaire des idées reçues en management (Eds, Pezet, A., Sponem, S.). Paris: La Découverte, 40-47.

- Piercy, N. F., Cravens, D. W., Lane, N., Vorhies, D. W. (2006). Driving Organizational Citizenship Behaviors and Salesperson - In-Role Behavior Performance: The Role of Management Control and Perceived Organizational Support. Journal of the Academy of Marketing Science 34 (2): 244-262.

- Piot, C., Kermiche, L. (2009). A quoi servent les comités d'audit? Un regard sur la recherché empirique. Comptabilité - Contrôle - Audit - Numéro thématique: 9-54. 
- Poksinska, B. (2007). Does Standardization have a Negative Impact on Working Conditions? Human Factors and Ergonomics in Manufacturing 17 (4): 383-394.

- Sampaio, P., Saraiva, P., Guimarães Rodrigues, A. (2009). ISO 9001 Certification Research: Questions, Answers and Approaches. International Journal of Quality \& Reliability Management 26 (1): 38-58.

- Savall, H., Zardet, V. (2005). Tétranormalisation : défis et dynamiques. Paris: Economica.

- Segrestin, D. (1996). La normalisation de la qualité et l'évolution de la relation de production. Revue d'Economie Industrielle - Numéro spécial Normalisation et organisation de l'industrie, 75: 291-307.

- Sharma, D. S. (2005). The Association between ISO 9000 Certification and Financial Performance. The International Journal of Accounting 40: 151-172.

- Sponem, S., Chatelain-Ponroy, S. (2013). Les comportements face au contrôle. In Contrôle de gestion - Perspectives stratégiques et managériales $-2^{e}$ édition (Eds, N. Berland, N., De Rongé, Y.). Paris: Pearson, 79-102.

- Sponem, S., Lambert, C. (2010). Pratiques budgétaires, rôles et critiques du budget Perception des DAF et des contrôleurs de gestion. Comptabilité - Contrôle - Audit 1: 159-194.

- Sun, H. (2000). Total Quality Management, ISO 9000 Certification and Performance Improvement. International Journal of Quality \& Reliability Management 17 (2): 168-179.

- Terziovski, M., Power, D., Sohal, A. S. (2003). The Longitudinal Effects of the ISO 9000 Certification Process on business Performance. European Journal of Operational Research 146: 580-595.

- Venkatesh, V., Morris, M. G., Davis, G. B., Davis, F. D. (2003). User Acceptance of Information Technology: Toward a Unified View. MIS Quaterly 27 (3): 425-478.

- Venkatesh, V., Davis, F. D. (2000). A Theoretical Extension of the Technology Acceptance Model: Four Longitudinal Field studies. Management Science 46 (2): 186-204.

- Véran, L. (2006). Activités et processus, modélisation gestionnaire et comportements des acteurs. Comptabilité - Contrôle - Audit 1: 65-84. 


\section{Annexes}

Annexe 1

\begin{tabular}{|c|c|c|c|c|}
\hline Nature & Hôpital privé & $\begin{array}{c}\text { Groupe de transport } \\
\text { routier de } \\
\text { marchandises }\end{array}$ & Chantier naval & $\begin{array}{l}\text { Entreprise de } \\
\text { chaudronnerie }\end{array}$ \\
\hline Activité & $\begin{array}{c}\text { Hôpital } \\
\text { pluridisciplinaire } \\
\text { (médecine, chirurgie, } \\
\text { obstétrique) }\end{array}$ & $\begin{array}{l}\text { Opérations de } \\
\text { transports, } \\
\text { d'organisation de } \\
\text { transports et de } \\
\text { logistique }\end{array}$ & $\begin{array}{c}\text { Construction de } \\
\text { navires militaires et } \\
\text { civils }\end{array}$ & $\begin{array}{l}\text { Fabrication de } \\
\text { composants } \\
\text { chaudronnés et } \\
\text { mécaniques }\end{array}$ \\
\hline Effectif approximatif & 500 & 1200 & 400 & 180 \\
\hline $\begin{array}{c}\text { Chiffre d'affaires } \\
\text { approximatif }\end{array}$ & $47 \mathrm{M} €$ & $200 \mathrm{M} €$ & $45 \mathrm{M} €$ & $35 \mathrm{M} €$ \\
\hline Périmètre certifié & $\begin{array}{l}\text { Le service } \\
\text { stérilisation }\end{array}$ & \multicolumn{3}{|c|}{ L'ensemble de l'organisation } \\
\hline
\end{tabular}

Caractéristiques des organisations constituant le terrain de l'étude

Annexe 2

\begin{tabular}{|c|c|c|c|c|c|}
\hline $\mathbf{N}^{\circ}$ & $\begin{array}{c}\text { Nature de } \\
\text { l'organisation }\end{array}$ & Fonction & Genre & $\begin{array}{c}\text { Année de } \\
\text { naissance }\end{array}$ & Statut \\
\hline 1 & Chantier naval & $\begin{array}{c}\text { Président directeur } \\
\text { général }\end{array}$ & Homme & 1955 & $\begin{array}{c}\text { Cadre } \\
\text { (supérieur) }\end{array}$ \\
\hline 2 & Hôpital & Pharmacien gérant & Femme & 1966 & $\begin{array}{c}\text { Cadre } \\
\text { (supérieur) }\end{array}$ \\
\hline 3 & Transport & Secrétaire général & Homme & 1967 & $\begin{array}{c}\text { Cadre } \\
\text { (supérieur) }\end{array}$ \\
\hline 4 & Hôpital & $\begin{array}{c}\text { Responsable du service } \\
\text { stérilisation }\end{array}$ & Femme & 1966 & Cadre \\
\hline 5 & Transport & $\begin{array}{c}\text { Adjoint au directeur } \\
\text { méthode et } \\
\text { développement }\end{array}$ & Homme & 1964 & Cadre \\
\hline 6 & Chantier naval & $\begin{array}{c}\text { Responsable du service } \\
\text { logistique }\end{array}$ & Homme & 1956 & Cadre \\
\hline 7 & Chantier naval & Ingénieur essais & Homme & 1985 & Cadre \\
\hline 8 & Chantier naval & Responsable coque & Homme & 1971 & Cadre \\
\hline 9 & Chantier naval & $\begin{array}{c}\text { Responsable de } \\
\text { production }\end{array}$ & Homme & 1955 & Cadre \\
\hline 10 & Hôpital & $\begin{array}{c}\text { Technicien de } \\
\text { maintenance }\end{array}$ & Homme & 1963 & Technicien \\
\hline 11 & Transport & $\begin{array}{c}\text { Responsable } \\
\text { réglementation }\end{array}$ & Homme & 1968 & $\begin{array}{c}\text { Technicien } \\
\text { (ETAM) }\end{array}$ \\
\hline 12 & Chaudronnerie & Chef d'atelier & Homme & 1984 & Technicien \\
\hline 13 & Chantier naval & $\begin{array}{c}\text { Dessinateur tuyauterie } \\
\text { en bureau d'études }\end{array}$ & Homme & 1965 & Technicien \\
\hline 14 & Chantier naval & Responsable tuyauterie & Homme & 1971 & Technicien \\
\hline 15 & Chantier naval & $\begin{array}{c}\text { Contremaître } \\
\text { chaudronnerie }\end{array}$ & Homme & 1973 & Technicien \\
\hline 16 & Chantier naval & Responsable secteur & Homme & 1973 & Technicien \\
\hline
\end{tabular}




\begin{tabular}{|c|c|c|c|c|c|}
\hline $\mathbf{N}^{\circ}$ & $\begin{array}{c}\text { Nature de } \\
\text { l'organisation }\end{array}$ & Fonction & Genre & $\begin{array}{c}\text { Année de } \\
\text { naissance }\end{array}$ & Statut \\
\hline 17 & Chantier naval & $\begin{array}{c}\text { Responsable supports à } \\
\text { la production }\end{array}$ & Homme & 1974 & Technicien \\
\hline 18 & Chantier naval & $\begin{array}{c}\text { Responsable secteur } \\
\text { montage-soudures }\end{array}$ & Homme & 1961 & Technicien \\
\hline 19 & Hôpital & Agent de stérilisation & Femme & 1965 & Agent \\
\hline 20 & Hôpital & Agent de stérilisation & Femme & 1970 & Agent \\
\hline 21 & Hôpital & Agent de stérilisation & Homme & 1967 & Agent \\
\hline 22 & Transport & Chauffeur routier & Homme & 1959 & Agent \\
\hline 23 & Transport & Chauffeur routier & Homme & 1958 & Agent \\
\hline 24 & Chantier naval & Contrôleur qualité & Homme & 1971 & Agent \\
\hline 26 & Chantier naval & Electricien de bord & Homme & 1977 & Agent \\
\hline 27 & Chaudronnerie & $\begin{array}{c}\text { Tourneur sur tour à } \\
\text { commande numérique }\end{array}$ & Homme & 1973 & Agent \\
\hline
\end{tabular}

\section{Caractéristiques des répondants}

\section{Notes}

${ }^{1}$ Huit principes fondent la norme ISO 9001 pour orienter l'attitude des salariés en faveur de la qualité : 1) Orientation client, 2) leadership, 3) Implication du personnel, 4) Approche processus, 5) Management par approche système, 6) Amélioration continue, 7) Approche factuelle pour la prise de décision, 8) Relations mutuelles bénéfiques avec les fournisseurs

${ }^{2}$ La fixation d'objectifs, la planification et le budget (phase Plan), la mise en œuvre (phase Do), le suivi des réalisations (phase Check), l'analyse des résultats et la prise d'actions correctives (phase Analyse) constituent le processus d'apprentissage du contrôle de gestion (Löning et al 2013).

${ }^{3}$ e.g. : marge bénéficiaire, croissance des ventes et bénéfice par action (Sharma 2005).

${ }^{4}$ Notamment l'implication du personnel, l'amélioration continue, le leadership et la satisfaction client.

${ }^{5}$ Selon le modèle d'Anderson et al. (1994), la satisfaction et l'engagement des employés (i.e. leur accomplissement) sont influencés par le management. L'accomplissement des employés s'inscrit dans le cadre d'un mécanisme de feedback agissant, entre autres, sur le leadership du management

${ }^{6}$ La tétranormalisation renvoie au quatre principaux pôles de normes qui s'exercent à l'entreprise, c'est-à-dire les normes du commerce international (e.g. les normes de l'OMC - Organisation Mondiale du Commerce), les normes comptables et financières (e.g. les normes IASB - International Accounting Standards Board), les normes d'assurance qualité, de sécurité et d'environnement (e.g. la norme ISO 9001) et les normes sociales (e.g. les normes du BIT - Bureau International du Travail), qui entrent en contradiction, affectant ainsi les règles du jeu économique et social de l'entreprise (Savall et Zardet 2005).

${ }^{7}$ A la différence d'une reconnaissance financière qui tend à diminuer la motivation intrinsèque, selon Deci (1971).

${ }^{8}$ Plus de 4600 entreprises employant presque 3 millions de salariés en 2010, selon les chiffres de l'Institut National de la Statistique et des Etudes Economiques (INSEE).

${ }^{9}$ " $\mathrm{QSR} \mathrm{N6}^{\odot}$ » est un logiciel d'analyse de données qualitatives. Ce logiciel permet l'indexation de données non numériques, non structurées. «QSR N6 ${ }^{\odot}$ " permet de coder des données qualitatives, c'est-à-dire de catégoriser des phénomènes (e.g. des variables d'attitude) à partir de documents écrits (e.g. les verbatim d'un entretien retranscrit).

10 «Decision Explorer $^{\circledR} »$ est un logiciel qui permet la représentation graphique de données textuelles et l'analyse de leur structure et de leur organisation (e.g. mesure de la centralité d'une variable dans un ensemble d'autres variables). Ce logiciel permet de repérer les concepts et leurs relations.

${ }^{11}$ i.e. des audits réciproquement réalisés par des intervenants extérieurs au secteur ou au poste concerné. 
${ }^{12}$ Le flicage s'apparente à une surveillance outrancière à des fins de sanction.

${ }^{13}$ Par exemple, l'analyse stratégique de Crozier et Friedberg (1977) supposant que, contrairement à l'idée selon laquelle il la subirait passivement, l'individu apparait en fait comme un acteur dans l'organisation disposant d'une relative liberté et qui agit à dessein dans l'organisation, cherchant à atteindre ses objectifs.

${ }^{14}$ Bien que les résultats de l'étude aient parallèlement fait émerger des croyances de qualification et de compétence, issues de la perception de la norme ISO 9001. 